\title{
From solar to stellar flare characteristics
}

\section{On a new peak size distribution for G-, K-, and M-dwarf star flares}

\author{
Konstantin Herbst ${ }^{1}$, Athanasios Papaioannou ${ }^{2}$, Saša Banjac ${ }^{1}$, and Bernd Heber ${ }^{1}$ \\ 1 Institut für Experimentelle und Angewandte Physik, Christian-Albrechts-Universität zu Kiel, 24118 Kiel, Germany \\ e-mail: herbst@physik.uni-kiel.de \\ ${ }^{2}$ Institute for Astronomy, Astrophysics, Space Applications and Remote Sensing (IAASARS), National Observatory of Athens, I. \\ Metaxa \& Vas. Pavlou St., 15236 Penteli, Greece
}

Received 6 February 2018 / Accepted 11 November 2018

\begin{abstract}
Context. The connection between solar energetic proton events and X-ray flares has been the focus of many studies over the past $13 \mathrm{yr}$. In the course of these investigations several peak size distribution functions based on Geostationary Operational Environmental Satellite (GOES) measurements of both quantities have been developed. In more recent studies one of these functions has been used to estimate the stellar proton fluence around the M-dwarf star AD Leonis. However, a comparison of the existing peak size distribution functions reveals strong discrepancies with respect to each other.

Aims. The aim of this paper is to derive a new peak size distribution function that can be utilized to give a more realistic estimate of the stellar proton flux of G-, K-, and M-dwarf stars.

Methods. By updating and extending the GOES-based peak size distribution down to B-class X-ray flare intensities with the help of SphinX data from the solar minimum conditions of 2009 and newly derived GOES data between 1975 and 2005, we developed a new power-law peak size distribution function for solar proton fluxes $(E>10 \mathrm{MeV})$. However, its resulting slope differs from values reported in the literature. Therefore, we also developed a double-power-law peak size distribution function. An extension to much higher X-ray flare intensities $\left(10^{-1}\right) \mathrm{W} \mathrm{m}^{-2}$ and above, for the first time, results in an approximation of best- and worst-case scenarios of the stellar proton flux around G-, K-, and M-dwarf stars.

Results. Investigating the impact of the newly developed peak size distribution function for G-, K-, and M-dwarf star flare intensities we show that in the worst-case scenario previous studies may underestimate the stellar proton flux by roughly one to five orders of magnitude.
\end{abstract}

Key words. Sun: flares - Sun: particle emission - Sun: X-rays, gamma rays - stars: flare

\section{Introduction}

Solar energetic particle (SEP) events originate from solar flares (SFs) and/or coronal mass ejections (CMEs) and are divided into two classes: impulsive and gradual. Whether an event is classified as impulsive or gradual strongly depends on its parent solar source (i.e., SF, CME; see Reames 1999, 2013, for further information). While impulsive SEP events are most likely associated with SFs (see, e.g., Klein \& Posner 2005) originating in resonant stochastic acceleration or magnetic reconnection (Aschwanden 2002) particles in gradual events may be accelerated by CMEdriven shocks (Reames 1999; Kahler 2001; Cane \& Lario 2006).

However, observations have indicated that these two categories are not able to cover the variety of the observed SEP event properties (Cane et al. 2010; Papaioannou et al. 2016), and that a third class of hybrid events exists during which both SFs and CME-driven shocks accelerate particles that contribute to large SEP events (see, e.g., Kocharov \& Torsti 2002; Kallenrode 2003). Although these hybrid events may look like gradual events they also show properties of impulsive events (see, e.g., Vainio et al. 2007), which may either result from acceleration of remnant ions at shock waves (Reames 2002) or from interactions of CMEs (Gopalswamy et al. 2002; Kahler \& Vourlidas 2014).

An armada of spacecraft widely distributed in heliolongitude have allowed the observation of SEP events filling a very broad region around the Sun. Thus, SEP events have been observed over a wide range of longitudes (see, e.g., Richardson et al. 2014; Dresing et al. 2014; Lario et al. 2016). Possible interpretations of the resulting widespread events include the propagation of CME-driven shocks that are able to inject SEPs over broad angular regions (Rouillard et al. 2012; Lario et al. 2016), crossfield diffusion (Dresing et al. 2012; Dröge et al. 2014), and/or the perpendicular transport of particles through processes other than diffusion (Wiedenbeck et al. 2012).

In order to decode the characteristics of SEP events it is necessary to establish empirical and/or semi-empirical statistical relations between the characteristics (e.g., peak flux, duration, fluence) of the SEP event and the observed properties of the parent solar events (see, e.g., Belov et al. 2005; Cliver et al. 2012; Papaioannou et al. 2016).

It has been shown that peak size distributions (PSDs) of solar flares that are associated with SEP events follow a power law: $f(x) \approx x^{-\alpha}$ (Hudson 1978; Belov et al. 2005). This representation of the relation between solar flares and SEP events points to the fact that X-ray and charged particle fluxes, originating from the same solar event, have an almost linear relation. These distribution functions depend on several different factors, for example the sample used in the analysis and the binning employed. This has led to differences of the derived slope (i.e., different 
$\alpha$ parameters) that consequently lead to differences in the expected $>10 \mathrm{MeV}$ peak proton flux values.

These relations also become important for studies of extrasolar environments, for instance the radiation environment around cool K- and M-dwarf stars. Because their habitable zones (HZs) are at small orbital separations a detection of orbiting planets within it becomes highly likely. Thus, these stars are favored targets to investigate Earth-like rocky exoplanets by spectroscopy, which will be the focus of future missions like the James Webb Space Telescope (JWST; see, e.g., Gardner et al. 2006) or the European Extremely Large Telescope (E-ELT; see, e.g., Gilmozzi \& Spyromilio 2007) with a focus on the search for biogenic molecules, the so-called biosignatures. Hence, in order to understand the upcoming measurements to the best extent, the effects of ultraviolet (UV) and extreme ultraviolet (EUV) radiation (spanning wavelengths from $124 \mathrm{~nm}$ down to $10 \mathrm{~nm}$ ) and stellar energetic particles on the atmospheric climate and chemistry have to be studied. This has been the focus of numerous recent studies that are set out to explore the habitability of exoplanets (see, e.g., Shields et al. 2016; Robinson 2017; Lingam \& Loeb 2017).

Although it is not possible to measure SEP-related stellar proton fluxes directly, a solar peak size distribution function might be used to quantify the expected radiation environment based on stellar X-rays (see, e.g., Segura et al. 2010). It should also be noted that stellar CMEs (Odert et al. 2017), the relation between CMEs-flares to other stellar systems (Moschou et al. 2017a,b), and the identification of the expected size of the exoplanetary magnetospheres (Patsourakos \& Georgoulis 2017) are currently under investigation by the scientific community and are evidence of the wealth of ideas and efforts that solar and space physics can bring to exoplanetary research. Our investigation was initiated by the identification of large differences between the published peak size distribution functions that have been widely used in the solar-space community. However, stellar and exoplanetary research efforts have also quite recently utilized PSDs, for example to estimate the impact of stellar cosmic rays on the exoplanetary atmospheric chemistry (see, e.g., Segura et al. 2010; Tilley et al. 2017). We will show that the conclusions drawn are subject to the choice of the PSD function.

In this study we first cross-compare and evaluate the different functions that are available in the literature (see Belov et al. 2005, 2007; Cliver et al. 2012, based on the $E>10 \mathrm{MeV}$ data measured by the Geostationary Operational Environmental Satellite, GOES), and then investigate the extention of the peak-size distributions of proton fluxes and associated soft $\mathrm{X}$-Ray flares from Q to $>\mathrm{X} 10$-class. To this end, we employ new data from the Solar Photometer in X-rays (SphinX) mission aboard the Complex Orbital Observations Near-Earth of Activity of the Sun-Photon (CORONAS-Photon) spacecraft (see Gryciuk et al. 2017) that measured the X-ray flux during the solar minimum of 2009, and also results from Cane et al. (2010).

Based on these measurements, we show that a new analytic distribution function is needed in order to properly describe the peak X-ray intensity to peak proton flux relationship for flares below the C-class flares. Furthermore, we use the proposed distribution function to describe not only the solar, but also the stellar soft X-ray peak-flux to proton peak-flux relationship of G-, K-, and M-dwarf stars. Our findings are compared to previous studies (see, e.g., Segura et al. 2010; Youngblood et al. 2016; Tilley et al. 2017) pointing towards a cross-quantification of the stellar proton fluxes and their corresponding fluences.

\section{Data used in the analysis}

\subsection{SphinX and its publicly available flare list}

SphinX, a spectrophotometer on board the CORONAS-Photon satellite (Kotov 2011), observed the solar soft X-ray emission during the period of extremely low solar activity of 2009 with a time resolution of 1-5 s (Sylwester et al. 2008, 2011, 2012, Gburek et al. 2011a,b, 2013; Kowalinski et al. 2012). The detectors observed the solar soft X-ray emission in an energy range similar to that of GOES (1-8 $\AA$ ) by covering $1.2-15 \mathrm{keV}$ (0.8-10 ̊). Launched on January 30, 2009, the spacecraft was put into a low polar orbit at an altitude of $550 \mathrm{~km}$ with an inclination of $82.5^{\circ}$. Although the mission was terminated unexpectedly at the end of 2009, SphinX almost continuously measured the X-ray flux between February 22 and November 29, 2009.

In 2009 SphinX was the only instrument that reliably measured the solar X-ray emission with high temporal and energy resolution. The sensitivity level of SphinX was thus almost a factor of 100 lower than can be achieved with GOES (around $3.73 \times 10^{-9} \mathrm{~W} \mathrm{~m}^{-2}$ ). This means that multiple events could be observed at levels never measured before. According to Gryciuk et al. (2017), within its nine-month operation more than $1600 \mathrm{X}$-ray flares were detected. In addition to the known flare classes (A, B, C, M, and X), which represent flux values between $1 \times 10^{-8}$ and $1 \times 10^{-4} \mathrm{~W} \mathrm{~m}^{-2}$ (see, e.g., Lang 2001), Gryciuk et al. (2017) introduced two new flare classes: $\mathrm{S}\left(\right.$ small $\left.=1 \times 10^{-9} \mathrm{~W} \mathrm{~m}^{-2}\right)$ and $\mathrm{Q}$ (quiet $=1 \times 10^{-10} \mathrm{~W} \mathrm{~m}^{-2}$ ).

Based on the SphinX flare list ${ }^{1}$, we generated a flare catalog of 93 Q-, 150 S-, 154 A-, 59 B-, and three C-class flares for the solar minimum of 2009 including the corresponding GOES proton fluxes ${ }^{2}$.

\subsection{Peak proton flux data}

In order to investigate the soft X-Ray flares and their associated proton peak-size distributions, we correlated the measured $\mathrm{X}$-ray fluxes to the $>10 \mathrm{MeV}$ integral proton fluxes that are calculated by the differential proton measurements ${ }^{3}$. These measurements are routinely made by the Energetic Particle Sensor (EPS), one of the four instruments of the Space Environment Monitor (SEM), on board GOES (Onsager et al. 1996).

Thus, we first retrieved the $5 \mathrm{~min}$ averaged integral proton flux $>10 \mathrm{MeV}$ data $^{4}$. The solar flares identified in the SphinX flare catalog did not result in a considerable enhancement in the integrated GOES proton fluxes above 10,50 , and $100 \mathrm{MeV}$. Therefore, we evaluated the background and standard deviation of the proton fluxes during a time interval of four hours after the soft X-ray peak for the selected flares. The upper limit of the GOES proton intensity was consequently set as three standard deviations above background (see Klein et al. 2010). As can be seen in Fig. 1, this results in a population with a mean proton flux value of $0.485 \mathrm{pfu}$ (dashed line). Additionally, we evaluated

\footnotetext{
1 http://156.17.94.1/sphinx_11_catalogue/SphinX_cat_ main.html

2 The flare list is available at http://www . ieap.uni-kiel.de/et/ ag-heber/SphinX

3 The differential proton flux at a set of different energy channels (ranges) per unit solid angle are measured in particles $/ \mathrm{cm}^{2} \mathrm{~s} \mathrm{sec} \mathrm{MeV}$. The integral proton fluxes utilized in our study result from the integration of the product of these differential proton fluxes and are given in particle flux units (pfu), i.e., particles $/ \mathrm{cm}^{2} \mathrm{~s} \mathrm{sec}$.

4 Data retrieved from https://satdat.ngdc.noaa.gov/sem/ goes/data/
} 


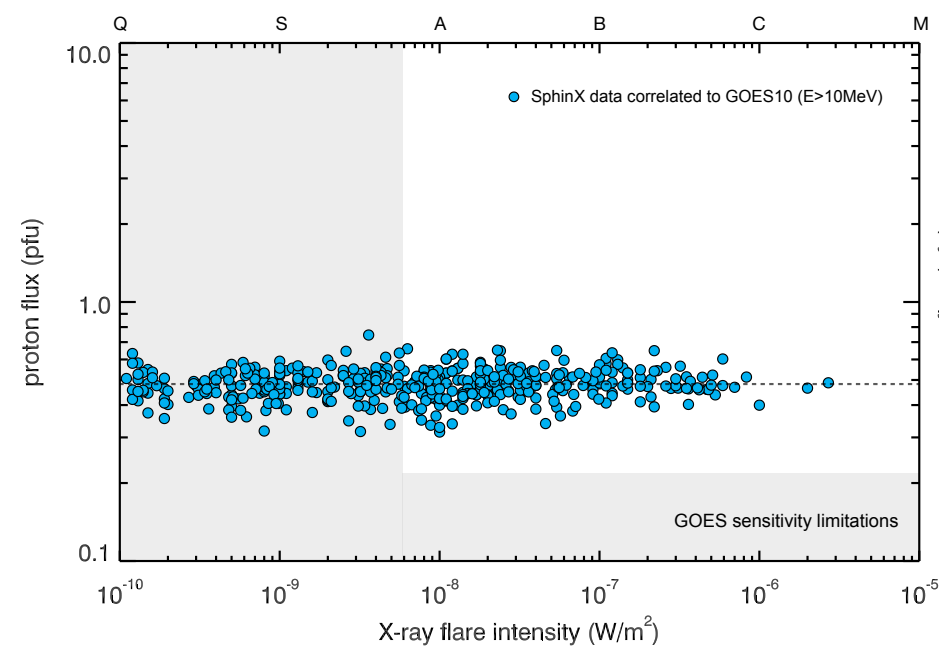

Fig. 1. Scatter plot between the measured X-ray fluxes of the SphinX instrument and the $>10 \mathrm{MeV}$ integral proton fluxes measured with the EPS aboard the GOES satellite. The shaded areas mark the GOES sensitivity limits of the X-ray flare intensities $\left(3.8 \times 10^{-9} \mathrm{~W} \mathrm{~m}^{-2}\right)$ and the corresponding peak proton fluxes $(0.22 \pm 0.08 \mathrm{pfu})$ for the year 2009 .

the sensitivity level of GOES for the same time period. Therefore, we used $5 \mathrm{~min}$ averaged data and calculated the observed mean flux (in pfu) for 2009, which led to a mean proton flux of $0.22 \pm 0.08 \mathrm{pfu}$. At the same time, taking into account that the uncertainty imposed by the GOES data is an important factor when looking for "clear" enhancements, we further evaluated the upper limits for different confidence intervals (i.e., 68\%, 99.5\%, and $99.75 \%$ ) of the peak proton flux for 2009 and obtained the corresponding upper thresholds of $0.31,0.44$, and $0.67 \mathrm{pfu}$, respectively.

\section{Size distributions of solar flares and solar energetic particle events}

Previously published studies on PSDs present the associated soft $\mathrm{X}$-ray peak fluxes of solar flares to the measured peak proton fluxes at $E>10 \mathrm{MeV}$. Taking into account the SphinX soft X-ray data, the previously published studies now can be extended down to B-class flares $\left(10^{-7} \mathrm{~W} \mathrm{~m}^{-2}\right)$, as shown in Fig. 2. We note, however, that each PSD depends on different samples. Therefore, we gathered all available samples and their corresponding PSDs. The first sample published in Belov et al. (2005) includes the averaged values that correspond to $\geq \mathrm{C}$-class solar flares located at W15-W75 (magenta dots). The second sample comes from Cliver et al. (2012) who presented west-limb flares from W20-W80 (black dots). The third sample by Papaioannou et al. (2016) used all $\geq \mathrm{C}$ class flares originating from the whole visible solar disk, i.e., E90-W90 (green dots). It is also worth noting that these studies used different methods (and samples) to produce the class-dependent values. In particular, Belov et al. (2005) used a catalog of 617 SEP events at $E>10 \mathrm{MeV}$, covering 28 years of observations, defined log-equal soft X-ray intensity intervals and calculated the corresponding mean value per bin. Expanding on this former approach, Belov et al. (2007) utilized an updated database of 673 SEP events and solar flares (with an identified association of 1:1) distributed over 31 years of measurements. At the same time Cliver et al. (2012), made use of 52 SEP events at $>10 \mathrm{MeV}$ that exceeded 1 pfu associated with $\geq$ M1.0 solar flares over a time period of nine years (their Fig. 1). Papaioannou et al. (2016), on the other hand, used a

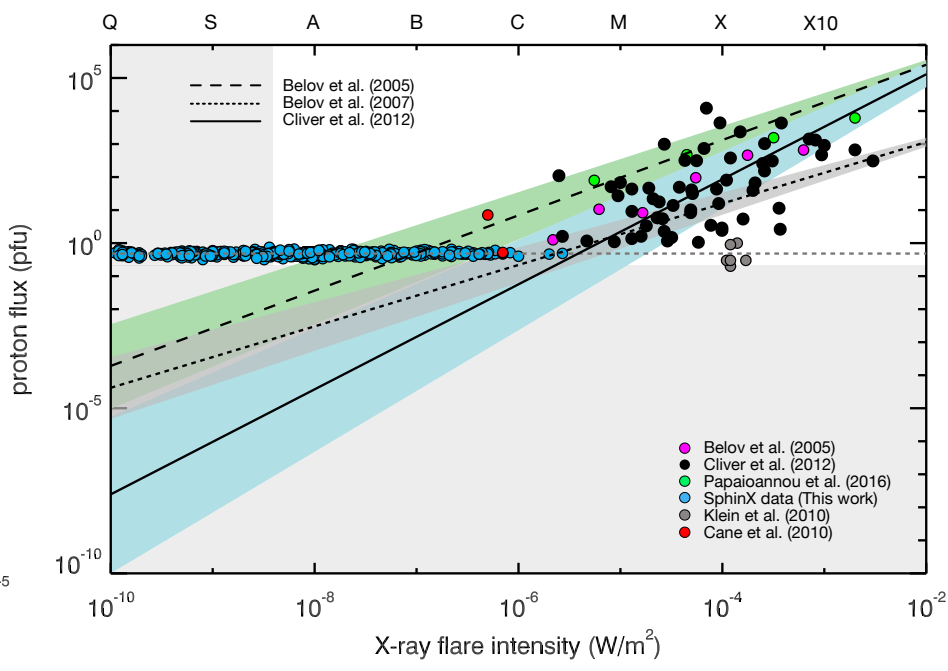

Fig. 2. Peak size distributions by Belov et al. (2005, 2007) and Cliver et al. (2012) with their respective errors, plotted on top of the data points gathered in this study. The shaded areas mark the GOES sensitivity limits shown in Fig. 1.

catalog of 314 SEP events, defined one bin per flare class (i.e., C, $\mathrm{M}, \mathrm{X},>\mathrm{X} 10$ ), and calculated the mean flare flux and peak proton flux for each bin (their Fig. 4).

Existing peak size distribution functions, however, are solely based on $\geq \mathrm{C}$-class solar flares. These functions, which are based on the peak X-ray flare intensity $I_{x}$, are given by Belov et al. (2005; based on W15-W75 flares):

$I_{\mathrm{p}}(E>10 \mathrm{MeV})=(4.8 \pm 1.3) \times 10^{7} \cdot I_{x}^{1.14 \pm 0.14} ;$

Belov et al. (2007; based on W20-W80 flares):

$I_{\mathrm{p}}(E>10 \mathrm{MeV})=(8.3 \pm 1.2) \times 10^{4} \cdot I_{x}^{0.93 \pm 0.10} ;$

Cliver et al. (2012; based on W20-W80 flares):

$I_{\mathrm{p}}(E>10 \mathrm{MeV})=1.96 \times 10^{8}\left( \pm 5.71 \times 10^{8}\right) \cdot I_{x}^{1.59 \pm 0.25}$.

A comparison of the previously discussed measurements with the PSDs by Belov et al. (2005, 2007) and Cliver et al. (2012) can also be found in Fig. 2. As can be seen, the distribution functions significantly differ from each other in the low and in the high X-ray flare intensity range. Furthermore, it becomes obvious that the PSD developed by Cliver et al. (2012) more closely fits the derived X-ray flare intensity to proton flux relation.

In order to quantify the differences in the expected peak proton flux based on different PSDs Table 1 provides the calculated values for C-, M-, and X-soft X-ray flare classes. It becomes obvious that with increasing $\mathrm{X}$-ray flare intensity the expected peak proton flux increases significantly for either of the PSDs. However, an inter-comparison of the model-dependent outputs shows large differences for all flare classes. Therefore, the strongest deviations can be found between Belov et al. (2005) and Belov et al. (2007). We note that, although often disputed, the PSD by Belov et al. (2007) is not a correction of the PSD given in Belov et al. (2005). Unfortunately, both functions are not able to reflect the corresponding GOES estimates (magenta dots).

Moreover, Cane et al. (2010) identified three B-class flare associated SEP events. Their published catalog includes 280 SEP events that extended to $>25 \mathrm{MeV}$ and occurred in the years 
Table 1. Expected peak proton fluxes (in pfu) for C-, M-, and X-class flares derived by different PSDs.

\begin{tabular}{cccc}
\hline \hline Reference & C & M & X \\
\hline Belov et al. 2005 & 43.46 & 600 & 8280.7 \\
Belov et al. 2007 & 0.97 & 8.3 & 70.65 \\
Cliver et al. 2012 & 0.73 & 28.42 & 1105.65 \\
\hline
\end{tabular}

1997-2006. These three events were marked on December 6, 1997 (as a B7.0-class flare), April 4, 2003 (as a B7.0-class flare), and November 7, 2003 (as a B5.0-class flare). For these events we were able to identify pronounced peaks for the events on April 7 and November 7, 2003. However, although labeled as a SEP event, we were not able to identify an enhancement above the background in the integral proton flux above $10 \mathrm{MeV}$ for the B-class flare related proton flux on December 6, 1997. For consistency, the expected $>10 \mathrm{MeV}$ peak flux values were calculated based on the same procedure applied to the SphinX soft X-ray measurements (see Sect. 2.2). As can be seen in Fig. 2, the three B-class related SEP events by Cane et al. (2010), given as red dots, fall well within the SphinX values.

Additionally, Klein et al. (2010) found seven SEP events in CME-less X-class flares from the western hemisphere. The corresponding $>10 \mathrm{MeV}$ values can be found in the second column of their Table 3. As can be seen, the corresponding peak protonflux values fall into the same level as the bulk of the soft X-ray flares identified with the SphinX data. The lowest values of this population, showing values about $2 \%$ below the mean values based on SphinX. In addition, the corresponding sensitivity of the GOES measurements (see Sect. 2.2) needs to be taken into account in order to identify the current limitations in measuring proton fluxes from lower class solar flares.

\section{Development of an updated size distribution function}

\subsection{Data}

For further elaboration of the B-class flare ensemble, we performed a systematic scan of the particle and soft X-ray GOES data between 1975 and 2005. Within this time span, we were able to identify eight SEP events associated with B-class flares, and one event associated with an A1.5 solar flare (Dr. Anatoly Belov, priv. comm.). To include these B-class flares into our study, we inspected the $>10 \mathrm{MeV}$ GOES proton data in the same manner as previously discussed. For all events a corresponding SEP event was found, the most pronounced of which occurred on April 24, 1999.

In addition, we reduced the number of events from the SphinX soft X-ray flare catalog to a total of 18 events by selecting only those events that could be allocated with magnetically wellconnected flare regions, i.e., within W20-W80. Furthermore, we inspected the time profiles of ions recorded by the Electron Proton Helium Instrument (EPHIN, Müller-Mellin et al. 1995) aboard the Solar and Heliospheric Observatory (SOHO) at an energy range of 4.3-25 MeV for these events. We note that EPHIN is ten times more sensitive than GOES (see, e.g., Posner 2007). Furthermore, we also inspected the electron recordings by the Electron, Proton, and Alpha Monitor (EPAM, Gold et al. 1998) aboard the Advanced Composition Explorer (ACE), at an energy range of $0.038-0.053 \mathrm{MeV}$. However, none of these solar flares resulted in any signature in the SOHO/EPHIN and ACE/EPAM channels.
At the same time, the peak proton flux of the B-class related SEP events identified by Cane et al. (2010), match the 18 wellconnected SphinX measurements and the nine A-/B-class flares found in the GOES measurements. Applying the upper GOES threshold of $0.67 \mathrm{pfu}$ for the peak proton flux (see Sect. 2.2) in total 13 enhancements above the $99.75 \%$ GOES sensitivity level can be utilized for further studies. Based on these new events a "traditional" power-law reflecting the low and high X-ray flare intensity relationship can be found (see Sect. 4.2). However, this proved to be flatter than recently proposed power laws.

Therefore, in order to mathematically describe all populations as well as possible, a new double-power-law PSD function is also proposed.

\subsection{Development of an updated size distribution function}

In order to develop a function that is able to reflect the peak size distribution of $\mathrm{B}$ - to $>\mathrm{X} 10$-class flares, the following steps were performed:

1. We first applied a traditional power-law function based on a reduced major axis (RMA) $)^{5}$ regression, as suggested by Cliver et al. (2012). To this end, we employed all data points, including the 13 enhancements in the $<\mathrm{C}$-class flares regime, 1 event from Cane et al. (2010), and 1 SphinX event that exceeded the $99.7 \%$ GOES sensitivity threshold. Based on this method we found the best fit peak size distribution function to be in the form of

$I_{p}(E>10 \mathrm{MeV})=6.63 \times 10^{5}\left( \pm 1.842 \times 10^{5}\right) \cdot I_{x}^{0.954 \pm 0.17}$.

The result is shown in panel 1 of Fig. 3 as a red curve together with its corresponding error-band. As can be seen, this power law is able to reflect the low-intensity range fairly well. However, the differences above M-class flares become stronger with increasing intensity. At the same time the resulting $\gamma$ of 0.954 differs from the value reported in the literature (Belov et al. 2005, 2007; Cliver et al. 2012), which falls at $\approx 1.2$. Moreover, recent studies obtained a $\gamma$ of 1.59 for the SEP proton flux of a sample of $>$ C2.0 class flares (Cliver et al. 2012) and concluded that the differences in the $\gamma$ for PSDs of SEPs and SXRs arise primarily because the flares that lead to SEP events constitute a special, energetic subset of all flares, that is further associated with fast CMEs (Cliver et al. 2012; Cliver \& D'Huys 2018). However, it should be noted that the PSDs of flare electromagnetic emissions present a $\gamma \sim 1.7$ for hard X-rays and microwaves (see Table 1 in Cliver et al. 2012).

2. Although a $\gamma$ of $\sim 1.2$ seems to be quite explanatory of the SEP proton flux distributions over several orders of magnitude (i.e., >C2.0 class flares; Belov et al. 2007; Cliver \& D'Huys 2018) it is not representative of the flare electromagnetic emissions (Cliver et al. 2012). Therefore, given the even flatter $\gamma$ obtained with the RMA-based power law (e.g., 0.954; when considering all $>$ B-class flares) and taking into account the possibility that a subset of the size distribution of all SEP flares will not follow a power law (see Fig. 3 in Belov et al. 2007), we studied the intersection of the mean $<$ C-class population $(6.919 \mathrm{pfu})$ and the RMA-based power-law function given in Eq. (4). As can be seen in panel 2 of Fig. 3 this intersection can be found at an intensity of $6.04 \times 10^{-6} \mathrm{~W} \mathrm{~m}^{-2}$ (i.e., C6.0 class flares).

\footnotetext{
The RMA regression accounts for uncertainties in the $x$ and $y$ directions. Other then the ordinary least-squares (OLS) fit method the RMA minimizes the sum of the vertical and horizontal distances of the data points from the resulting fit function by minimizing the areas of the triangles formed by the observations and the regression line.
} 


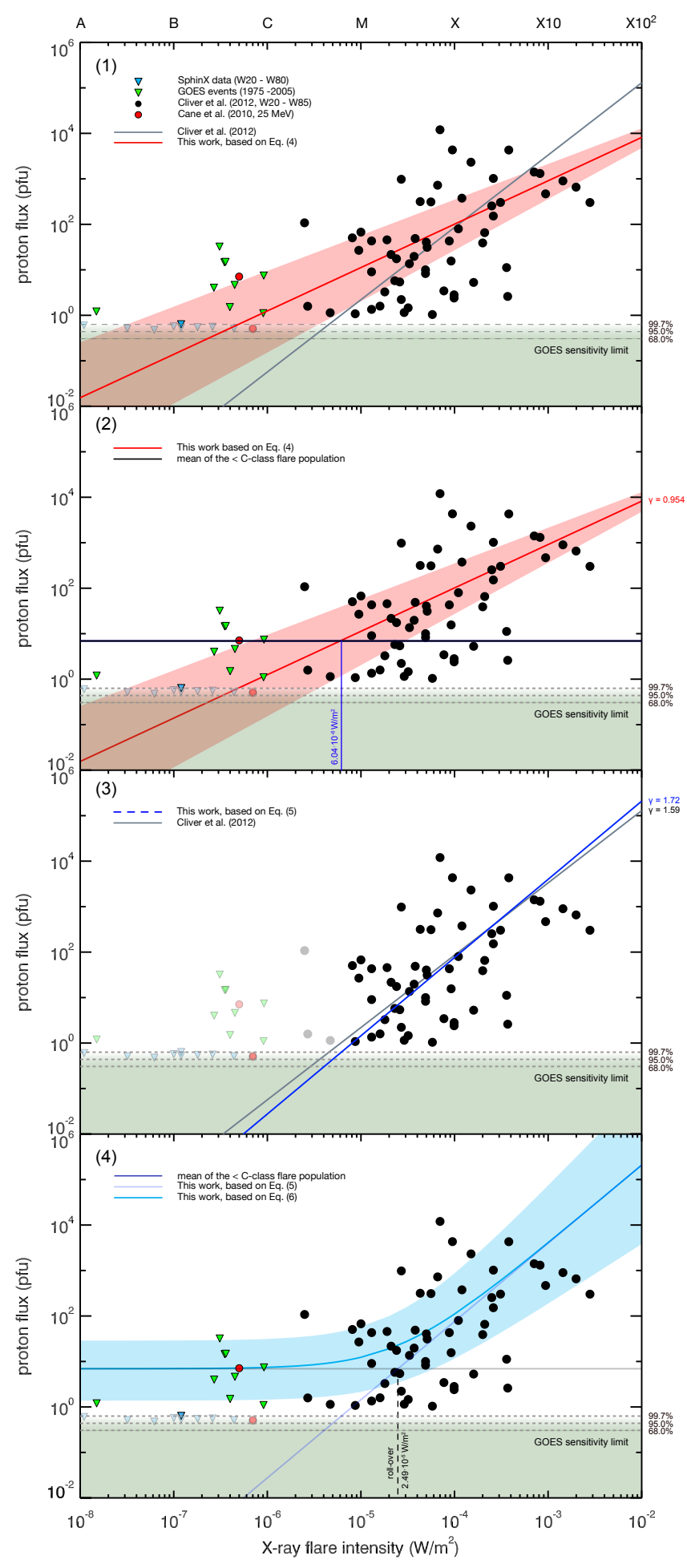

Fig. 3. Development of the new size distribution function, as described in Sect. 4.2. Panel $a$ refers to step (2), panel $b$ to step (3), and panel $c$ to step (4).

3. Building on this, we excluded all data points below this intersection intensity and applied the RMA method to the remaining population ( $>$ C6.0 class). As a result, a similar PSD to Cliver et al. (2012; gray line, $\gamma=1.59$ ) was identified, although with an even steeper slope of $\gamma=1.72$ (blue line), as can be seen in panel 3 of Fig. 3. The resulting power law has the form $I_{p}(E>10 \mathrm{MeV})=1.96 \times 10^{8}\left( \pm 5.75 \times 10^{8}\right) \cdot I_{x}^{1.72 \pm 0.397}$.
4. Finally, in order to mathematically reflect the plateaulike population for $<\mathrm{C}$-class flares and the steeper rise in the $>$ C-class regime as closely as possible, an exponential function with an X-ray flare intensity-dependent roll-over was employed. Therefore, both the mean value of the $<\mathrm{C}$ - class flares and the slope of $1.72 \pm 0.39$ are used as fixed input parameter. The resulting PSD function has the form

$I_{p}(E>10 \mathrm{MeV})=\left(a \cdot I_{x}+\left(b \cdot \exp \left(-0.001 \cdot I_{x}\right)\right)^{\gamma}\right.$,

with $a=1.22 \times 10^{5}\left( \pm 7.17 \times 10^{4}\right), b=3.05( \pm 1.79)$, and $\gamma=1.72 \pm 0.397$. The results are displayed as a blue curve in panel 4 of Fig. 3. As can be seen, this function (a) is able to reflect the events that were associated with lower energy soft X-ray flares (i.e., A- to C-class flares), (b) fits well the population of C-class flares and above, and (c) results in a roll-over around M2.5 class flares. To provide an error estimate to this peak size distribution function the scipy.optimize.leastsq fitting routine $^{6}$ has been applied. Thus, the Jacobian matrix is multiplied with the residual variances, estimated by the mean square errors. The resulting covariance matrix then is used to derive the standard error and, therefore, the $\pm \sigma$ uncertainty. The $\pm \sigma$ uncertainties are displayed as light blue bands in Figs. 3 (lower panel), 4 (lower right panel), and 5 .

\subsection{Validity of the PSDs}

To test the validity of the newly derived PSD functions, and to compare it to the PSDs discussed in Sect. 3, we compared the theoretical values with measured $E>10 \mathrm{MeV}$ peak proton fluxes corresponding to ground level enhancement (GLE) events, strong SEP events that can be measured at the Earth's surface. Therefore, we used the GLE list given by Belov et al. (2009 their Table 1). The results of this comparison are given in Fig. 4. However, since then two more GLEs (GLE71 and GLE72) have occurred. Therefore, we inspected the GOES measurements and expanded the catalog by Belov et al. (2009) accordingly. Thus, in Fig. 4 the black and red dots correspond to the actual $(E>$ $10 \mathrm{MeV}$ ) peak proton fluxes of the GLEs, while lines represent the calculated values of the PSD functions given in Eqs. (1)-(5) based on the associated solar flare intensities. Also given are the upper and lower limits according to the estimated errors as error-bands. As discussed previously (see, e.g., Fig. 2), not all PSD functions are able to reflect the measurements. In particular, Belov et al. (2007) (upper right panel of Fig. 4) is out of scope for almost all X-ray flare classes. Surprisingly, Belov et al. (2005) fits well to the low-intensity flares (C-class), while being out of scope for GLEs associated to X-ray flares above C-class (upper left panel of Fig. 4). The lower left panel shows the PSD proposed by Cliver et al. (2012). This function is clearly able to represent very well the high-intensity flares while being out of scope for the $<$ C-class flares. Additionally, the lower right panel of Fig. 4 depicts the double power law with the roll-over (in blue) as well as the RMA power law for solar flares above B-class (in red).

As can be seen, Eq. (4) represents quite well the relation between the magnitude of solar flares and the corresponding peak proton flux, although it is not descriptive of the resulting peak proton flux for stronger flares (e.g., >X1.0 class). However, it provides a better identification in low-energy flares compared to the other PSDs (Belov et al. 2005, 2007; Cliver et al. 2012).

6 Provided at https://docs.scipy.org/doc/scipy-0.19.0/ reference/generated/scipy.optimize.leastsq.html 

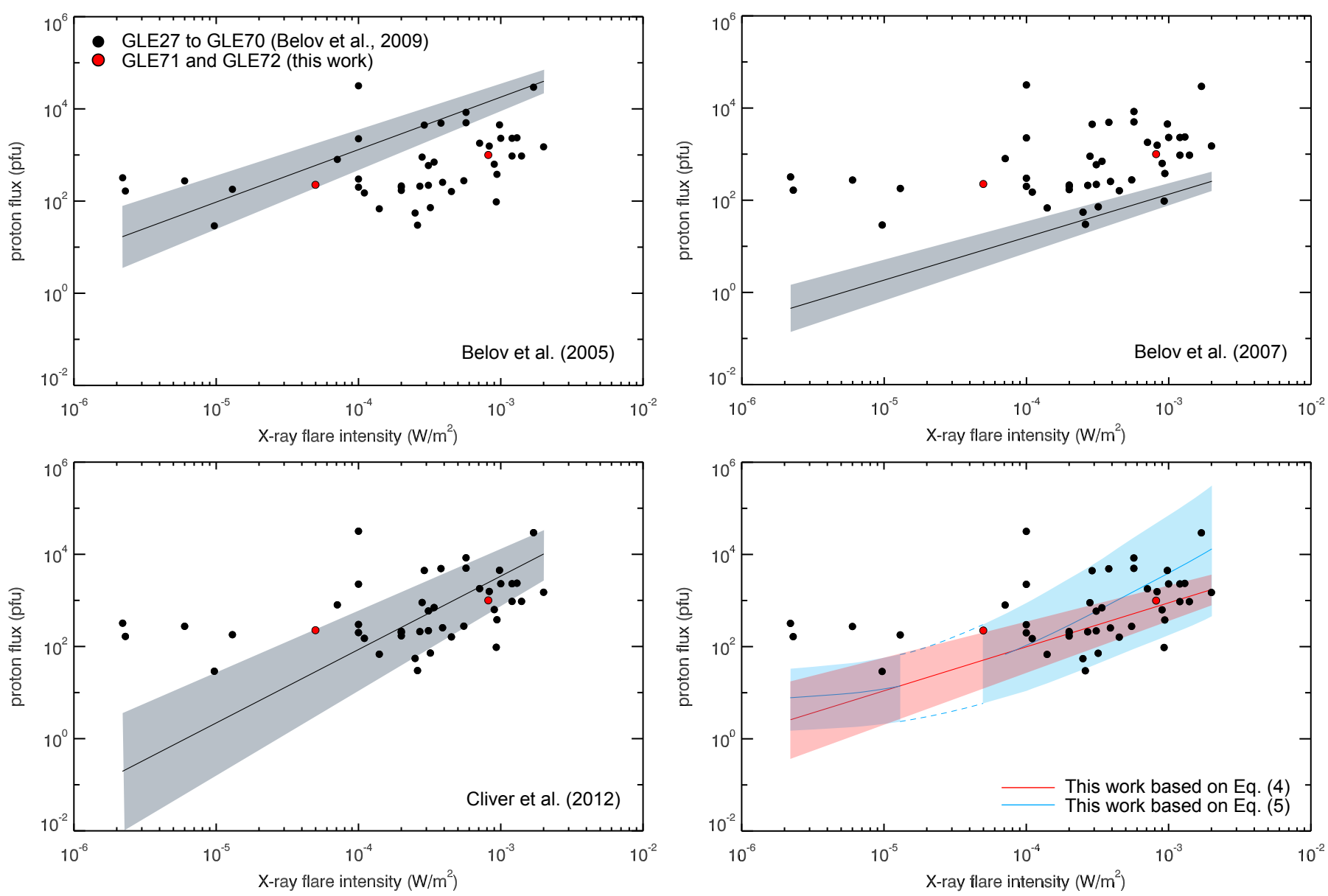

Fig. 4. Comparison of the different PSD functions with respect to the GOES measurements during the Ground level enhancements 27-72. Upper left panel: Belov et al. (2005). Upper right panel: Belov et al. (2007). Lower left panel: Cliver et al. (2012). Lower right panel: this work.

At the same time, Eq. (5) provides comparable results for $<\mathrm{X}$ class flares, and contrary to Eq. (4) better quantifies the relation between the X-ray flare intensity and the expected proton peak flux for more intense flares.

\section{From solar to stellar flare characteristics}

Belov et al. (2005) showed that with increasing X-ray flare intensity it becomes more likely to detect proton events, supporting the hypothesis that proton acceleration takes place in the same active region in which the X-ray flares are produced. In the case of G-, K-, and M-dwarf stars proton events cannot be measured in situ. However, observations have shown that solar X-ray flares and M-dwarf star flares follow the same fundamental relationships (see, e.g., Butler et al. 1988). Thus, the measured stellar X-ray flare intensities can be used as a proxy for the proton fluxes of the most intensive flares. However, it should be noted that there are several factors that enhance the uncertainty when extrapolating PSDs up to the upper limit of solar flares. In particular, although solar flares and CMEs are closely associated with SEP events (Reames 1999), the exact underlying physical mechanisms that play a role at each SEP event are still rather unclear (Cane et al. 2010). In addition, there are only a handful of solar flares that have been recorded at the Sun with a class of $>X 10$, and thus there seems to be an upper limit for SXRs. This is partially attributed to the GOES SXR saturation around $10^{-3} \mathrm{~W} \mathrm{~m}^{-2}$ (Youngblood et al. 2016), but also to the power-law frequency distribution of SXR solar flares (Veronig et al. 2002). As a result, the expected peak proton flux of an SEP event at such a highenergy flare regime, is practically unpredictable. Additionally, as shown in this work, the differences between the peak size distribution functions available in literature (discussed in Sects. 3 and 4) lead to strong deviations when extrapolated to X-ray flare intensities of G-, K-, and M-dwarf stars, i.e., $>10^{-1} \mathrm{~W} \mathrm{~m}^{-2}$.

Based on the above, it is also important to seek for the expected upper limit of soft X-ray fluxes. It is currently known that the X28 solar flare recorded on November 4, 2003, is the largest solar flare observed in soft X-rays to date. Based on this observational evidence an upper limit for the energy of solar flares at the Sun was found to be $\sim 6 \times 10^{33} \mathrm{erg}$ (Aulanier et al. 2013), corresponding to an upper flare flux of $\sim$ X700 (0.07 W m ${ }^{-2}$, see Cliver \& Dietrich 2013).

At the same time, an independent study noted that if for solar-like stars the X-ray emission is comprised of solar flares and assuming that a scaling between solar flares and CMEs holds, then such solar flare-CME relations cannot be extrapolated to arbitrarily high flare energies, and thus may flatten off around $\geq 10^{31} \mathrm{erg}$, corresponding to an upper flare flux of $\geq X 1$ $\left(10^{-4} \mathrm{~W} \mathrm{~m}^{-2}\right.$, see Drake et al. 2013).

Furthermore, using Kepler data Shibayama et al. (2013) investigated superflares on solar-type stars. Among other things, they found that most active G-type dwarfs are able to produce flare energies above $10^{36} \mathrm{erg}$ (see also Davenport 2016). We note that Shibayama et al. (2013) estimated the total uncertainty in flare energy to be $\pm 60 \%$. Nevertheless, with this result the upper limit of X-ray flare intensities of active Sun-like stars is above 
K. Herbst et al.: From solar to stellar flare characteristics

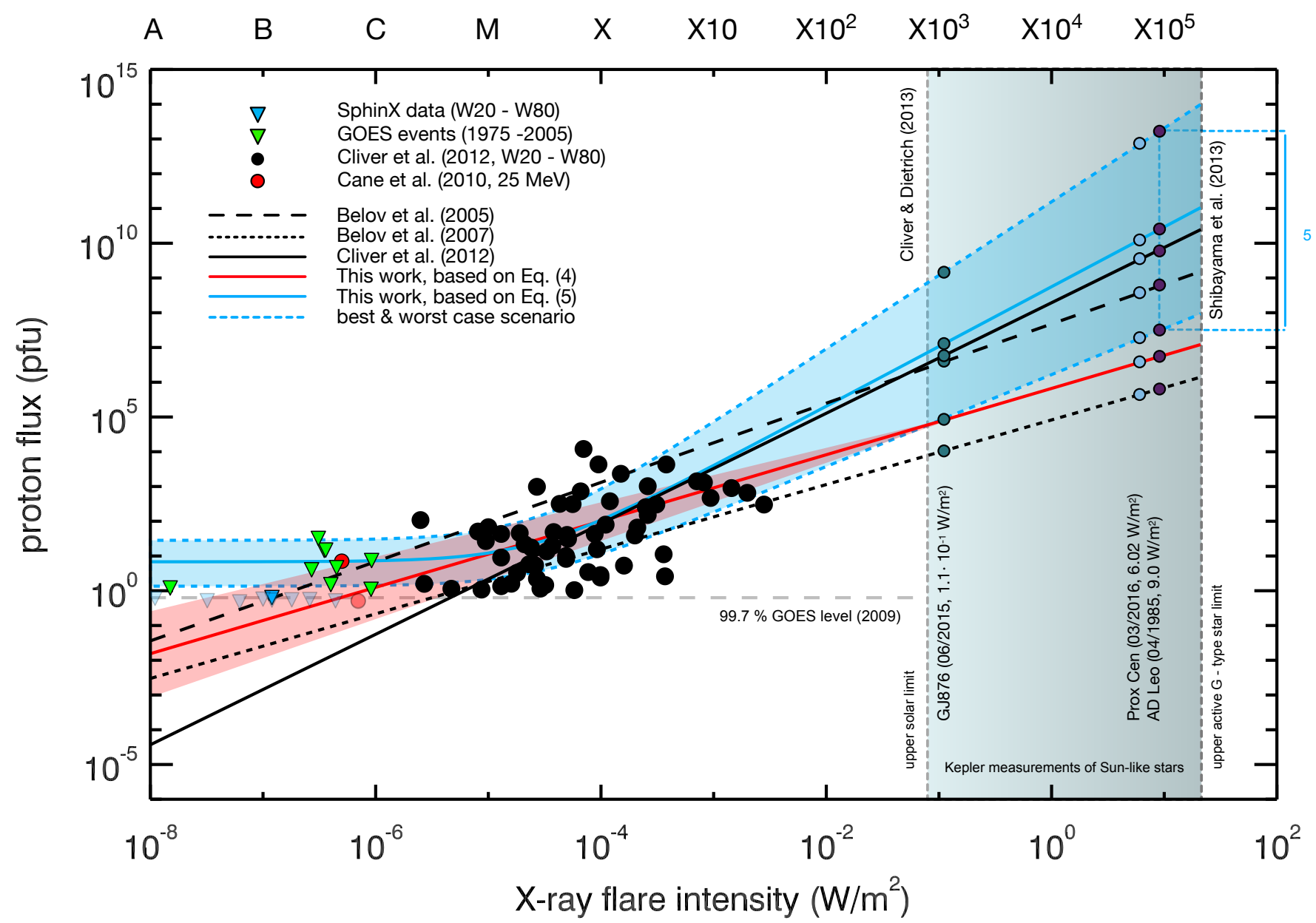

Fig. 5. Estimation of the proton flux around G-, K-, and M-dwarf stars based on the size distribution functions available in the literature (black lines) and proposed in this work (colored lines). Black dots represent GOES measurements of W20-W80 flare events according to Cliver et al. (2012), red dots represent three events listed in Cane et al. (2010), green triangles show the $<$ C-class flare GOES events found between 1975 and 2005, and the blue triangles display the well-connected SphinX events. Due to the sensitivity limitations of GOES (i.e., 0.67 pfu according to the 99.7\% limit, dashed line) only fully colored data points have been taken into account. The gray shaded box represents the X-ray flare intensity range between the upper solar limit proposed by Cliver \& Dietrich (2013) and the upper limit of active G-type stars observed with Kepler and proposed by Shibayama et al. (2013). In addition, the corresponding X-ray flare intensity to proton flux values of the M-dwarf star flare events measured at GJ876 $\left(I_{x}=1.1 \times 10^{-1} \mathrm{~W} \mathrm{~m}^{-2}\right.$, see Youngblood et al. 2016, green dots $)$, Proxima Centauri $\left(I_{x}=6.02 \mathrm{~W} \mathrm{~m}^{-2}\right.$, see Howard et al. 2018, blue dots) and AD Leo $\left(I_{x}=9 \mathrm{~W} \mathrm{~m}^{-2}\right.$, see Segura et al. 2010, purple dots) are displayed. For the last case, the best- and worst-case scenario proposed in this work allow for peak proton flux values between $3.03 \times 10^{7}$ and $1.63 \times 10^{13} \mathrm{~cm}^{-2} \mathrm{sr}^{-1} \mathrm{~s}^{-1}$.

$10 \mathrm{~W} \mathrm{~m}^{-2}$. Moreover, investigating the activity of 540 flaring Mdwarf stars in the Kepler field, Yang et al. (2017) found the upper flare energy limit to be on the order of $2 \times 10^{35} \mathrm{erg}$ (see their Fig. 7), which is well within the upper active G-type star limit.

However, according to Vidotto et al. (2016), among others, fast-rotating M-dwarf stars might have surface magnetic fields that are orders of magnitude stronger than observed at the Sun. Because of these strong magnetic fields stellar eruptions might be confined, and thus an acceleration of particles could become impossible (see, e.g., Wang \& Zhang 2007; Schrijver 2009; Drake et al. 2013). Nonetheless, the need to identify the particle radiation environment at G-, K-, and M-dwarf stars warrants an extrapolation of PSDs to the super-flare regime, even though it should be treated with caution (see also Youngblood et al. 2016).

One of the most observed M-dwarf systems is AD Leonis (AD Leo, G1388), a M3.5V star located at a distance of $4.9 \mathrm{pc}$ from the Sun. According to Scalo et al. (2007), its ratio of soft $\mathrm{X}$-ray to bolometric luminosity, which can be seen as a measure of its stellar activity, is $10^{3}$ times higher than the activity of the Sun. Segura et al. (2010) studied the proton fluence of a stellar flare event which occurred on April 12, 1985, on AD Leo (see, e.g., Hawley \& Pettersen 1991) to investigate its influence on the planetary atmospheric enhancement of $\mathrm{N}, \mathrm{NO}$, and $\mathrm{NO}_{2}$ as well as $\mathrm{H}, \mathrm{OH}$, and $\mathrm{H}_{2} \mathrm{O}$ in the upper strato- and mesosphere and the resulting ozone depletion. According to Thomas et al. (2007) and Rodger et al. (2008), the 1859 Carrington solar proton event may have lead to a local $\mathrm{O}_{3}$ depletion of $\sim 10 \%$. This solar proton event had a calculated fluence of $\sim 10^{10} \mathrm{~cm}^{2}$ for protons with energies $>30 \mathrm{MeV}$, which is roughly four times larger than the solar proton fluence of the February 1956 event, the largest event of the modern era (Rodger et al. 2008).

Figure 5 shows the extrapolation of the proton flux to X-ray flare intensities of G-, K-, and M-dwarf stars, which is assumed to be within the upper solar and active G-type like star limitations $\left(10^{-1}-10 \mathrm{~W} \mathrm{~m}^{-2}\right)$, based on the peak size distribution functions by Belov et al. (2005; solid line), Belov et al. (2007; dotted line), and Cliver et al. (2012; dashed line). In addition the extrapolation due to Eq. (4) and (5) is shown as a red and blue line, respectively. It is obvious that the different slopes of the PSD functions (i.e., $\gamma=1.59$ according to Cliver et al. 2012, while 
Table 2. Estimated proton fluxes and fluences of the AD Leo flare event on April 12, 1985, according to the different PSDs and Carrington event fluences.

\begin{tabular}{|c|c|c|c|c|c|c|c|}
\hline & \multicolumn{3}{|c|}{ Previous studies } & \multicolumn{4}{|c|}{ This work } \\
\hline & BE05 & BE07 & CL12 & RMA & Best case & DPL mean & Worst case \\
\hline $\mathrm{d} F / \mathrm{d} t\left(\mathrm{~cm}^{-2} \mathrm{sr}^{-1} \mathrm{~s}^{-1}\right)$ & $5.88 \times 10^{8}$ & $6.41 \times 10^{5}$ & $6.45 \times 10^{9}$ & $5.39 \times 10^{6}$ & $3.03 \times 10^{7}$ & $2.45 \times 10^{10}$ & $1.63 \times 10^{13}$ \\
\hline$F\left(\mathrm{~cm}^{-2}\right)$ & $1.50 \times 10^{12}$ & $1.63 \times 10^{9}$ & $1.64 \times 10^{13}$ & $1.38 \times 10^{10}$ & $7.73 \times 10^{10}$ & $6.26 \times 10^{13}$ & $4.17 \times 10^{16}$ \\
\hline$F_{\text {ADLeo }} / F_{\text {Carr,SE10 }}$ & 200 & 0.22 & 2187 & 1.83 & 10.31 & 8340 & $5.55 \times 10^{6}$ \\
\hline$F_{\mathrm{ADLeo}} / \mathrm{F}_{\mathrm{Carr}, \mathrm{CL} 13}$ & 136 & 0.15 & 1491 & 1.25 & 7 & 5687 & $3.79 \times 10^{6}$ \\
\hline
\end{tabular}

Notes. Shown are the values based on the PSDs by Belov et al. (2005 denoted BE05), Belov et al. (2007 denoted BE07), Cliver et al. (2012) (denoted CL12), the RMA-based function given in Eq. (4) (denoted RMA), and the double power-law function given in Eq. (5).

$\gamma=0.945$ according to Eq. (4) and $\gamma=1.72$ according to Eq. (5)) result in differences in the high X-ray flare intensity regimes.

However, as observed at the Sun, a certain variety of the $\mathrm{X}$-ray flare intensity-dependent peak proton flux values is expected. As can be seen, our double-power-law function is the first PSD function that provides an error estimate of the expected peak proton fluxes for X-ray flares $>10^{-1} \mathrm{~W} \mathrm{~m}^{-2}$. Therefore, both the lower and upper level, represent extreme cases of the X-ray flare intensity-dependent peak proton fluxes that can be expected around G-, K-, and M-dwarf stars, and therefore in the following are denoted best-case and worst-case scenario, respectively. It becomes obvious, that for superflares the expected peak proton flux values can differ by up to seven orders of magnitude.

Segura et al. (2010) used different scaling relations for M-dwarf stars utilizing the broadband near-UV and the $1-8 \AA$ flare flux based on Mitra-Kraev et al. (2005) to estimate the X-ray flare intensity for the flare event of April 1985 observed on AD Leo. Based on the estimated X-ray flare intensity value of $9 \mathrm{~W} \mathrm{~m}^{-2}$, they applied the PSD function by Belov et al. (2005) and calculated the corresponding peak proton flux of $5.9 \times$ $10^{8} \mathrm{~cm}^{-2} \mathrm{sr}^{-1} \mathrm{~s}^{-1}$ for protons with $E>10 \mathrm{MeV}$. They concluded that the event fluence was on the order of 200 times higher than the fluence of the Carrington event $\left(7.5 \times 10^{9} \mathrm{~cm}^{-2}\right.$, see Segura et al. 2010).

Utilizing the calculated $\mathrm{X}$-ray flare intensity of $9 \mathrm{~W} \mathrm{~m}^{-2}$ reveals differences of around seven orders of magnitude with respect to the values based on the PSD by Belov et al. (2007) and our proposed worst-case scenario. The PSD-dependent corresponding peak proton flux values, denoted $\mathrm{d} F / \mathrm{d} t$, are given in the first row of Table 2. As can be seen, the estimated peak proton flux by Segura et al. (2010) is well within our best- and worstcase scenario. While overestimating the event-dependent peak proton flux by a factor of 50 compared to the best-case scenario, the resulting flux could also have been more than three orders of magnitude higher.

Furthermore, we calculated the PSD-function dependent proton fluences (denoted $F$, second row of Table 2) and compared these values to one of the strongest solar energetic particle events known to mankind, the Carrington event from 1859, to which a proton fluence of $7.5 \times 10^{9}$ protons $\mathrm{cm}^{-2}$ has been attributed (see Segura et al. 2010). Our study, however, shows that the flare likely produced a proton event roughly 42 times stronger, which results in a fluence on the order of 10 to $6 \times 10^{6}$ times that during the Carrington event. Moreover, newer studies revised the Carrington event fluence to $1.1 \times 10^{10}$ protons $\mathrm{cm}^{-2}$ (see Cliver $\&$ Dietrich 2013). The updated $F_{\text {ADLeo }} / F_{\text {Carr }}$ values are given in the last row of Table 2 . As can be seen, this reduces the $F_{\text {ADLeo }} / F_{\text {Carr }}$ values by about $32 \%$.
Based on the fact that the AD Leo event had a 200 times higher fluence than the Carrington event, Segura et al. (2010) concluded that, for oxygen-rich Earth-like planets in the habitable zone of an active M- dwarf star, a stellar proton event like this would lead to a $94 \%$ depletion of ozone inside the exoplanetary atmosphere. As a consequence, the atmosphere would need decades to recover from such an event.

However, our study shows that an event such as the one at ADLeo could result in an event 10 to $10^{7}$ times stronger than the Carrington event. Therefore, the best-case scenario would result in proton events comparable to those detected in terrestrial cosmogenic radionuclide records, for example at 774/5AD and 993/4AD (see, e.g., Miyake et al. 2012, 2013; Mekhaldi et al. 2015), whereas for the worst-case scenario the ozone depletion due to such an event would be likely even stronger than $94 \%$, which then most likely would also extend the atmospheric recovery time.

In addition, we studied two more measured flare events around the M-dwarf stars GJ876 $\left(I_{x}=1.1 \times 10^{-1} \mathrm{~W} \mathrm{~m}^{-2}\right.$, see Youngblood et al. 2016) and Proxima Centauri $\left(I_{x}=6.02 \mathrm{~W} \mathrm{~m}^{-2}\right.$, see Howard et al. 2018). A comparison of the PSD-dependent peak proton flux values is given in Table 3. Leaving aside the results based on Belov et al. (2007), it shows that the proton flux values only differ slightly from each other in the case of the GJ876 X-ray flare event of June 2015. However, due to the higher X-ray flare intensity and the difference in the slopes of the PSD functions, the results for the Proxima Centauri flare, measured in March 2016, show differences almost as large as those for the AD Leo event.

Nevertheless, it should be kept in mind that according to Grenfell et al. (2012) major Earth-like events on AD Leo occur about once every eight hours, which is faster than the recovery time for ozone and $\mathrm{NO}_{x}$. Furthermore, the results by Shibayama et al. (2013) suggest that some G-type stars could have even stronger flares (i.e., super- or hyperflares) that can occur once every 10 days.

Thus, although potential life on Earth-like exoplanets may not be directly affected by such strong events, it should be kept in mind that there is a direct influence on the detectability of the habitability of exoplanets. Because such strong events will lead to variations in the (exo)planetary ion-chemistry, it is conceivable that an interpretation of future (exo)planetary atmospheric measurements may be misleading.

\section{Summary and conclusions}

The motivation of this study was to evaluate the widely used peak size distribution functions (PSDs) of the soft X-ray flares 
Table 3. Estimated proton fluxes in pfu of observed stellar flares of the M-dwarf stars GJ876 (observed in June $2015, I_{X}=1.1 \times 10^{-1} \mathrm{~W} \mathrm{~m}{ }^{-2}$, see Youngblood et al. 2016), Proxima Centauri (observed in March 2016, $I_{X}=6.02 \mathrm{~W} \mathrm{~m}^{-2}$, see Howard et al. 2018), and AD Leonis (observed in April 1989, $I_{X}=9 \mathrm{~W} \mathrm{~m}^{-2}$, see Segura et al. 2010).

\begin{tabular}{|c|c|c|c|c|c|c|c|}
\hline & \multicolumn{3}{|c|}{ Previous studies } & \multicolumn{4}{|c|}{ This work } \\
\hline & BE05 & BE07 & CL12 & RMA & Best case & DPL mean & Worst case \\
\hline GJ876 & $3.88 \times 10^{6}$ & $1.10 \times 10^{4}$ & $5.86 \times 10^{6}$ & $8.07 \times 10^{4}$ & $8.93 \times 10^{4}$ & $1.26 \times 10^{7}$ & $1.45 \times 10^{9}$ \\
\hline Proxima Centauri & $3.72 \times 10^{8}$ & $4.41 \times 10^{5}$ & $3.40 \times 10^{9}$ & $3.67 \times 10^{6}$ & $1.78 \times 10^{7}$ & $1.23 \times 10^{10}$ & $6.97 \times 10^{12}$ \\
\hline ADLeonis & $5.88 \times 10^{8}$ & $6.41 \times 10^{5}$ & $6.45 \times 10^{9}$ & $5.39 \times 10^{6}$ & $3.03 \times 10^{7}$ & $2.45 \times 10^{10}$ & $1.63 \times 10^{13}$ \\
\hline
\end{tabular}

and peak proton fluxes from the solar and the stellar scientific communities.

To this end we cross-compared the published results from Belov et al. (2005, 2007) and Cliver et al. (2012) and marked their differences (see Fig. 2). Furthermore, we employed new data from the SphinX instrument, which measured the soft $\mathrm{X}$-ray flux during the solar minimum conditions in 2009. Utilizing these measurements we attempted to extended the flare range of the PSDs up to B-class flares (see Fig. 3).

However, by taking into account SEP events associated with B-class flares, the resulting proton fluxes from well-connected flares identified by SphinX, and by applying an upper threshold of $0.67 \mathrm{pfu}(99.7 \%)$ for the sensitivity of GOES measurements, we first attempted to derive a "typical" PSD utilizing a simple RMA power law. However, this led to a $\gamma$ of 0.954 and underestimated the expected peak proton flux for stronger flares (see Fig. 3 and Fig. 4). Nonetheless, it was shown that a simple power law represents very well the population $>\mathrm{C} 2.0$ flares Cliver et al. (2012) and >C6.0 flares (this work).

Equation (4) represents a power law in the SEP proton fluxes that for the first time takes into account $<\mathrm{C}$-class flares. However, the resulting $\gamma$ of 0.945 differs significantly from those reported in the literature (Belov et al. 2005, 2007; Cliver et al. 2012).

Moreover, we proposed a new peak size distribution function in the form similar to a double-power-law function (see, e.g., Band et al. 1993) with an M-class flare intensity dependent rollover at around $2.5 \times 10^{-5} \mathrm{~W} \mathrm{~m}^{-2}$ (M2.5 flares, see Eq. (5) and Fig. 3) in order to reflect the plateau-like B-class population as well as possible.

It should also be noted that based on the upper flare flux of active G-type stars ( $10^{36} \mathrm{erg}$; see Shibayama et al. 2013) and the corresponding estimated flare flux of the AD Leo event of $I_{X}=$ $9 \mathrm{~W} \mathrm{~m}^{-2}$ (Segura et al. 2010), which is well within the limitations, the PSDs presented in this work are in reasonable agreement with the expected high flare energies. Nonetheless, it seems that an upper limit in the high-energy flare regime is inevitable, since the peak flux of solar flares follows a power law with an index of $\sim 2$, as shown by data (Veronig et al. 2002; Yashiro et al. 2006; Cliver et al. 2012) and theory (Aschwanden \& Freeland 2012), consequently constraining the related peak proton fluxes.

In order to estimate the stellar proton flux around G-, K-, and M-dwarf stars, PSD functions have to be extended to flares with intensities well above X10 values. Segura et al. (2010) and Tilley et al. (2017) have thus based their estimates for the proton flux around flare-rich M-dwarf stars on the PDS function by Belov et al. (2005). A comparison of the G-, K-, and M-dwarf star peak X-ray flare intensity to peak proton flux correlation based on the PDS functions by Belov et al. (2005, 2007), Cliver et al. (2012), the RMA-based PSD function proposed in Eq. (4), as well as the double power-law function given in Eq. (5) showed strong differences of up to seven orders of magnitude (see Fig. 5). These discrepancies are also reflected when the stellar proton fluence is calculated (see Tables 2 and 3). Based on our findings we were able to show that an AD Leo-like flare can result in proton events on the order of 10 to $10^{7}$ times stronger than the Carrington event. While the first can be compared to the extreme solar events recorded in terrestrial cosmogenic radionuclide records (Miyake et al. 2012, 2013; Mekhaldi et al. 2015) the latter would have dramatic consequences for Earth-like exoplanets in the habitable zone of such flaring stars.

As a result Segura et al. (2010), who found the fluence to be 200 times higher then the Carrington event, concluded that i) such a strong stellar proton event would lead to a $94 \%$ depletion of ozone inside the exoplanetary atmosphere of an oxygen-rich Earth-like planets in the habitable zone of an active M- dwarf star, and ii) that it would take decades for the atmosphere to recover from the event. While our best-case scenario (lower limit Eq. (5)) would most likely lead to a much weaker depletion of ozone, the ozone depletion due to the April 1985 event would likely be even stronger than $94 \%$, which probably would also extend the atmospheric recovery time when the proton flux of our worst-case scenario is applicable. It is also worth noting that such strong (or even stronger events) are not as rare as one might think. According to Grenfell et al. (2012) major Earth-like events on AD Leo occur about once every eight hours, which is faster than the recovery time for ozone and NOx. Furthermore, Shibayama et al. (2013) suggest that some G-type stars could have even stronger flares (super- or hyperflares) that can occur once every ten days. However, among other things, this strongly depends on how the fluence is distributed over the energy of the primary CR particles. Thus, knowing the stellar radiation environment, and in turn being able to model the radiation exposure on the surface of an Earth-like exoplanet, is crucial in order to assess its habitability and has to be studied in much more detail in the future.

Undoubtedly there are several uncertainties when trying to quantify the radiation environment at Earth-like exoplanets. First, scaling-laws derived from measurements of our star need to be used; the Sun (which provides an upper limit for the corresponding flare flux, orders of magnitude less than is expected in stellar environments) (see Fig. 5). However, this is a necessary first step in working towards this problem (Segura et al. 2010; Youngblood et al. 2016). Second, adding to this uncertainty, more sensitive measurements at the low-energy flare regime are necessary in order to clearly extend the PSDs. Finally, the discrepancy between the slopes of the size distributions of proton flares and the general population of SXR flares is still under debate. In this work, we summarized these uncertainties and their effects, utilizing all observational data at hand. Additionally, we derived a new traditional PSD and further proposed a mathematical double-power-law PSD, both of which are able 
to reflect the relation between the flux of solar flares and the expected peak proton flux to some extent. Finally, we quantified the effect of the use of these PSDs to the exoplanetary environment for three known (published) cases (see Fig. 5 and Table 3). Therefore, this work provides the current known limitations for both the solar and stellar physics community with respect to the employed scaling laws and further shows the necessity for more sensitive measurements both in the low- and the high-energy flare regime.

Acknowledgements. We thank the SphinX collaboration for providing the $\mathrm{X}$-ray intensity profiles, the flare catalog, and the flare list via http://156. 17.94.1/sphinx_11_catalogue/SphinX_cat_main.html, as well as the GOES collaboration for providing their data via https://satdat.ngdc. noaa.gov/sem/goes/data/. KH and AP would like to thank Anatoly Belov, Ed Cliver, and Ludwig Klein for stimulating discussions. SB thanks the German Research Foundation, DFG, for financial support via the project The Influence of Cosmic Rays on Exoplanetary Atmospheric Biosignatures (Project number 282759267). KH, SB, and BH would also like to thank M. Sinnhuber and V. Schmidt (Karlsruhe Institute of Technology), as well as H. Rauer, J. L. Grenfell, and M. Scheucher from the Deutsches Zentrum für Luft- und Raumfahrt (DLR) Berlin and the Technical University of Berlin.

\section{References}

Aschwanden, M. J. 2002, Space Sci. Rev., 101, 91

Aschwanden, M. J., \& Freeland, S. L. 2012, ApJ, 754, 112

Aulanier, G., Démoulin, P., Schrijver, C., et al. 2013, A\&A, 549, A66

Band, D., Matteson, J., Ford, L., et al. 1993, ApJ, 413, 281

Belov, A., Garcia, H., Kurt, V., \& Mavromichalaki, E. 2005, Cosmic Res., 43, 171

Belov, A., Kurt, V., Mavromichalaki, H., \& Gerontidou, M. 2007, Sol. Phys., 246, 457

Belov, A. V., Erosehnko, E. A., Kryakunova, O. N., Kurt, V. G., \& Yanke, V. G. 2009, Proceedings of the 31st ICRC, 2976

Butler, C.J., Rodonó, M., \& Foing, B. J. 1988, A\&A, 206, L1

Cane, H., \& Lario, D. 2006, Space Sci. Rev., 123, 45

Cane, H. V., Richardson, I. G., \& von Rosenvinge, T. T. 2010, J. Geophys. Res., 115,1

Cliver, E. W., \& D'Huys, E. 2018, ApJ, 864, 48

Cliver, E. W., \& Dietrich, W. F. 2013, J. Space Weather Space Clim., 3, A31

Cliver, E. W., Ling, A. G., Belov, A., \& Yashiro, S. 2012, ApJ, 756, L29

Davenport, J. R. A. 2016, ApJ, 829, 23

Drake, J. J., Cohen, O., Yashiro, S., \& Gopalswamy, N. 2013, ApJ, 764, 170

Dresing, N., Gomez-Herrero, R., Klassen, A., et al. 2012, Sol. Phys., 281, 281

Dresing, N., Gómez-Herrero, R., Heber, B., et al. 2014, A\&A, 567, A27

Dröge, W., Kartavykh, Y., Dresing, N., Heber, B., \& Klassen, A. 2014, J. Geophys. Res., 119, 6074

Gardner, J. P., Mather, J. C., Clampin, M., et al. 2006, Space Sci. Rev., 123, 485 Gburek, S., Siarkowski, M., Kepa, A., et al. 2011a, Sol. Syst. Res., 45, 182

Gburek, S., Sylwester, J., Kowalinski, M., et al. 2011b, Sol. Syst. Res., 45, 182

Gburek, S., Sylwester, J., \& Kowalinski, E. A. 2013, Sol. Phys, 283, 631

Gilmozzi, R., \& Spyromilio, J. 2007, The Messenger, 127, 3

Gold, R., Krimigis, S., Hawkins, S., et al. 1998, The Advanced Composition Explorer Mission (Berlin: Springer), 541

Gopalswamy, N., Yashiro, S., Michalek, G., et al. 2002, ApJ, 572, L103

Grenfell, J. L., Grießmeier, J.-M., von Paris, P., et al. 2012, Astrobiology, 12, 1109

Gryciuk, M., Siarkowski, M., Sylwester, J., et al. 2017, Sol. Phys., 292, 77

Hawley, S. L., \& Pettersen, B. R. 1991, ApJ, 378, 725

Howard, W. S., Tilley, M. A., Corbett, H., et al. 2018, ApJ, 860, L30

Hudson, H. 1978, Sol. Phys., 57, 237
Kahler, S. W. 2001, J. Geophys. Res., 106, 20947

Kahler, S. W., \& Vourlidas, A. 2014, ApJ, 784, 47

Kallenrode, M.-B. 2003, J. Phys. G: Nucl. Part. Phys., 29, 965

Klein, K. L., Posner, A., et al. 2005, A\&A, 438, 1029

Klein, K.-L., Trottet, G., \& Klassen, A. 2010, Sol. Phys., 263, 185

Kocharov, L., \& Torsti, J. 2002, Sol. Phys., 207, 149

Kotov, Y. 2011, Syst. Res., 45, 153

Kowalinski, M. 2012, in Photonics Applications in Astronomy, Communications, Industry, and High-Energy Physics, ed. R. Romaniuk, Proc. SPIE, 8454

Lang, K. R. 2001, The Cambridge Encyclopedia of the Sun (Cambridge, UK: Cambridge University Press)

Lario, D., Kwon, R.-Y., Vourlidas, A., Raouafi, N., \& Haggerty, D. 2016, ApJ, 819,72

Lingam, M., \& Loeb, A. 2017, Int. J. Astrobiol., 17, 116

Mekhaldi, F., Muscheler, R., Adolphi, F., et al. 2015, Nat. Commun., 6, 8611

Mitra-Kraev, U., Harra, L. K., Güdel, M., et al. 2005, A\&A, 431, 679

Miyake, F., Masuda, K., \& Nakamura, T. 2013, Nat. Commun., 4, 1748

Miyake, F., Nagaya, K., Masuda, K., \& Nakamura, T. 2012, Nature, 486, 240

Moschou, S. P., Drake, J. J., Cohen, O., et al. 2017a, ApJ, 850, 191

Moschou, S. P., Drake, J. J., \& Cohen, O. 2017b, BAAS, 49, 202.11

Müller-Mellin, R., Kunow, H., Fleißner, V., et al. 1995, Sol. Phys., 162, 483

Odert, P., Leitzinger, M., Hanslmeier, A., \& Lammer, H. 2017, MNRAS, 472, 876

Onsager, T., Grubb, R., Kunches, J., et al. 1996, Int. Soc. Opt. Photonics, 2812, 281

Papaioannou, A., Sandberg, I., Anastasiadis, A., Kouloumvakos, A., \& Georgoulis, M. 2016, J. Space Weather Space Clim., 6, A42

Patsourakos, S., \& Georgoulis, M. 2017, Sol. Phys., 292, 89

Posner, A. 2007, Space Weather, 5, 0500

Reames, D. V. 1999, Space Sci., Rev, 90

Reames, D. V. 2002, ApJ, 571, L63

Reames, D. V. 2013, Space Sci., Rev, 175

Richardson, I., von Rosenvinge, T., Cane, H., et al. 2014, Sol. Phys., 289, 3059

Robinson, T. D. 2017, in Handbook of Exoplanets, eds. H. J. Deeg, \& J. A Belmonte (Springer Living Reference Work), 67

Rodger, C. J., Verronen, P. T., Clilverd, M. A., Seppälä, A., \& Turunen, E. 2008, J. Geophys. Res.: Atmos., 113, D23302

Rouillard, A., Sheeley, N., Tylka, A., Vourlidas, A., Ng, C., et al. 2012, ApJ, 752, 44

Scalo, J., Kaltenegger, L., Segura, A. G., et al. 2007, Astrobiology, 7, 85

Schrijver, C. J. 2009, Adv. Space Res., 43, 739

Segura, A., Walkoicz, L. M., Meadows, V., Kasting, J., \& Hawley, S. 2010, Astrobiology, 10, 751

Shibayama, T., Maehara, H., Notsu, S., et al. 2013, ApJS, 209, 5

Shields, A. L., Ballard, S., \& Johnson, J. A. 2016, Phys. Rep., 663,

Sylwester, J., Kuzin, S., Kotov, Y., Farnik, F., \& Reale, F. 2008, JApA, 29, 339

Sylwester, B., Sylwester, J., Siarkowski, M., Engell, A. J., \& Kuzin, S. 2011, Cent. Eur. Astrophys. Bull, 35, 171

Sylwester, J., Kowalinski, M., \& Gburek, S. 2012, ApJ, 751, 111

Thomas, B. C., Jackman, C. H., \& Melott, A. L. 2007, Geophys. Res. Lett., 34, L06810

Tilley, M. A., Segura, A., Meadows, V. S., Hawley, S., \& Davenport, J. 2017, Astrobiology, submitted, [arXiv:1711.08484]

Vainio, R., Agueda, N., Aran, A., \& Lario, D. 2007, Space Weather: Research Towards Applications in Europe (Dordrecht, The Netherlands: Springer)

Veronig, A., Temmer, M., Hanslmeier, A., Otruba, W., \& Messerotti, M. 2002. A\&A, 382, 1070

Vidotto, A. A., Donati, J.-F., Jardine, M., et al. 2016, MNRAS, 455, L52

Wang, Y., \& Zhang, J. 2007, ApJ, 665, 1428

Wiedenbeck, M., Cohen, C., Nitta, N., Gomez-Herrero, R., \& Haggerty, D. 2012, ApJ, 762, 54

Yang, H., Liu, J., Gao, Q., et al. 2017, ApJ, 849, 36

Yashiro, S., Akiyama, S., Gopalswamy, N., \& Howard, R. 2006, ApJ, 650, L143

Youngblood, A., France, K., Loyd, R. P., et al. 2016, ApJ, 824, 101 